\title{
EFECTO DEL NITRÓGENO Y LA DENSIDAD DE POBLACIÓN EN EL CONTENIDO DE LISINA EN LA SEMILLA DE MAÍZ EN MÉXICO ${ }^{1}$
}

\author{
Mariano Mendoza-Elos ${ }^{2}$,Nicolás Moran-Vázquez²,Enrique Andrio-Enríquez $z^{2}$,Alfonso López-Benítez , Sergio A. \\ Rodríguez-Herrera ${ }^{3}$, Guillermo Castañón-Nájera ${ }^{4}$
}

\section{RESUMEN}

Efecto del nitrógeno y la densidad de población en el contenido de lisina en la semilla de maíz en México. El objetivo del presente trabajo fue identificar la disponibilidad de lisina en cultivares de maíz y su relación con la fertilización nitrogenada y densidad de población. Para ello, se evaluaron variedades de grano blanco, amarillo, QPM's y el Opaco-2 en la Universidad Autónoma Agraria Antonio Narro (UAAAN), Coahuila, México, en el año 2005; el factor, densidad de población estuvo a tres niveles y la fertilización nitrogenada en cuatro dosis. Para la evaluación se empleó un método microbiológico (Schizophyllum commune) y a través de una escala se realizó la selección de bajo y alto contenido de lisina. En campo se consideró un diseño experimental de bloques completos al azar con arreglo de parcelas subdivididas. Para el laboratorio se aplicó un diseño completamente al azar con la prueba múltiple Duncan. Se encontró una composición de proteína en el germen y el endospermo que varió de $18,73 \%$ a $21,29 \%$ y $9,04 \%$ a $10,36 \%$, respectivamente. Para el cultivar Opaco-2, se encontró $35,1 \%$ de proteína en el germen y $60,7 \%$ en el endospermo. Los valores para lisina variaron del $17 \%$ en la variedad de grano blanco hasta el 58\% para el Opaco-2. El porcentaje de lisina fue fuertemente influenciado por el genotipo, la densidad de población y fertilización nitrogenada.

Palabras clave: Maíz (QPM), fertilización nitrogenada, densidad de población, Schizophyllum commune, lisina.

\begin{abstract}
Effect of nitrogen and plant population density on lysine in maize in México. This study was carried out to identify the availability of lysine in maize cultivars and its relationship to nitrogen fertilization and plant population density by means of S. commune. Maize varieties QPM, opaque-2, synthetic-white and yellow kernels were evaluated in the Universidad Autónoma Agraria Antonio Narro (UAAAN) Coahuila, México, in the 2005. There were three levels of plant population density and four for nitrogen fertilization. Kernel lysine content was determined utilizing a microbiologic method (Schizophyllum commune) and a comparative scale that permitted to select high and low lysine content cultivars. In the field experiment a complete block design was utilized and arranged as a split-plot design. In the laboratory experiments, a complete randomized design and multiple ranges Duncan's test were utilized. Lysine content was estimated to range from $18,73 \%$ to $21,29 \%$ and from $9,04 \%$ to $10,36 \%$ for embryo and endosperm, respectively. For cultivars Opaque-2, contained $35.1 \%$ germ protein and 60,7\% endosperm protein. Lysine contents varied from $17 \%$ in whitekernel varieties to $58 \%$ in Opaque-2 hybrid. Lysine content was highly affected by the genetic the structure of the individuals and environment factors, but mostly by plant population density and nitrogen fertilization.
\end{abstract}

Key words: Maize QPM, nitrogen fertilization, population density, Schizophyllum commune, lysine.

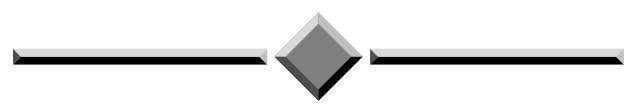

\section{INTRODUCCIÓN}

La tendencia en el consumo o ingesta de maíz es más elevada en las zonas rurales que en las urbanas, y en su producción, la zona subtropical de México aporta más del 30\% de la producción nacional de maíz. Al respecto, Chávez (1973) señala que en México gran parte del consumo nacional de calorías procede del maíz. En

\footnotetext{
Recibido: 19 de febrero, 2007. Aceptado: 29 de agosto, 2007. Presentado en la LII Reunión Anual del PCCMCA en Guatemala, Guatemala.

2 Instituto Tecnológico de Roque, Apdo. Postal 508, Km. 8 Carr. Celaya-Juventino Rosas, Roque, Celaya, Guanajuato, México CP 38110. Correo electrónico: mmendoza66@hotmail.com

3 Universidad Autónoma Agraria Antonio Narro, Departamento de Fitomejoramiento, Buenavista, Saltillo, Coahuila, México CP 25315;

4 Universidad Juárez Autónoma de Tabasco, 0.5 km Carret. Villahermosa Cárdenas, Villahermosa, Tabasco, México.
} 
las zonas rurales pobres, los hombres pueden consumir cerca de 600 g de maíz diarios, y las mujeres unos 400 g. CLARIDADES (2006) menciona que el consumo aparente per cápita de tortilla es de 179 g/día con una aportación a la nutrición de $32 \%$ a $55 \%$ de proteína.

Es muy importante considerar la calidad nutritiva del maíz, ya que son varios los años donde los centros de investigación, a través de los programas de mejoramiento genético, han tratado de incrementar la cantidad (y con menos interés) se han dedicado a la calidad de las proteínas. Existe un sin número de estudios sobre la composición química del maíz; en múltiples investigaciones que tratan de comprender y evaluar las repercusiones de la estructura genética del número elevado de variedades ancestrales y mejoradas, asimismo, la influencia de los factores ambientales, las prácticas agronómicas en los elementos constitutivos químicos y en el valor nutritivo del grano y sus partes anatómicas.

En México existe una gran diversidad genética de maíz, y los diversos programas de mejoramiento nacional e internacional constantemente liberan materiales, no obstante, el valor agregado de la proteína no se ha considerado hasta hoy como de impacto a la nutrición, por lo tanto, es necesario un análisis de los maíces de alto contenido de lisina de aspecto modificado y normal.

La lisina, uno de los aminoácidos limitantes en la calidad de la proteína, ha recibido una atención prioritaria para su identificación y cuantificación. Para ello, se han presentado diversos métodos biológicos, como alternativa ventajosa sobre los métodos químicos. Entre los métodos biológicos, los microbiológicos, basados en la respuesta de crecimiento de un microorganismo o por la cantidad de biomasa seca del mismo, en relación a la presencia del aminoácido, han cobrado gran interés y son una metodología necesaria para evaluar la calidad nutrimental del maíz QPM's y otros.

La importancia de los métodos microbiológicos en la determinación de aminoácidos, vitaminas y otros compuestos, ha cobrado gran interés por el tamaño de muestra, ahorro de tiempo en los análisis y su alta confiabilidad al compararlos con los métodos químicos. Schizophyllum commune, ha sido derivado de una mutación que se conoce como Mutación Letal
Condicional, que en ausencia del nutriente requerido, el mutante no puede crecer y moriría. Al mutante nutricional que tiene un requerimiento para un factor de crecimiento, como por ejemplo, algún aminoácido, vitamina ó ácidos orgánicos se denomina con el nombre de auxótrofos y aquellas células que los originan protótrofos (Wall y Paulis 1985).

La utilización de hongos y bacterias en los ensayos microbiológicos se basa en la gran variedad de substancias que estimulan o inhiben su crecimiento. Los microorganismos auxótrofos dependientes de aminoácidos dan una respuesta lineal al crecer en un medio de ensayo. Así, diversos investigadores han utilizado hongos y bacterias para la cuantificación de aminoácidos. Uno de ellos es Schizophyllum commune M-99, el cual es un hongo mutante para lisina (Wall y Paulis 1985).

El ciclo de vida, patrón base de sexualidad y forma de reproducción de Schizophyllum son los típicos grupos de hongos superiores clasificados dentro de los Himenomycetes. Es un hongo con micelios en forma de cetas, queda clasificado dentro de la familia Schizophyllaceae de los Polyporales, el género Schizophyllum. S. commune tiene su mayor distribución en zonas tropicales generalmente como parásito o saprófito en plantas leñosas, ocasionalmente en herbáceas (Raper y Milles 1958).

Los mismos autores señalan que el género difiere de otros, debido a que sus micelios están formados por dos láminas lisas, las cuales se arrizan separadamente una de otra bajo condiciones de desecación, difiere también en el crecimiento periferial e indeterminado de su cuerpo fructífero, éste desarrolla una diminuta concavidad lineal con elementos esporulantes que más tarde brotan, generando nuevamente micelios. Cada espora bajo condiciones apropiadas genera un micelio primario capaz de tener un crecimiento vegetativo indefinido, de este primer micelio se genera un segundo micelio que también puede tener una propagación vegetativa indefinida, durante la cual cada célula terminal se divide sincronizadamente (división conjugada) dando origen a células hijas (Raper y Milles 1958).

El cultivo y mantenimiento de cepas de Schizophyllum es simple y difieren solamente en detalle de las prácticas rutinarias con otros hongos filamentosos. 
El crecimiento fototrópico del microorganismo es de 0,5 a $0,7 \mathrm{~cm}$ por día a $23{ }^{\circ} \mathrm{C}$ y a $32{ }^{\circ} \mathrm{C}$ se duplica y a $15{ }^{\circ} \mathrm{C}$ disminuye a la mitad. Las cepas permanecen viables por varios años sobre medio completo a $1-4{ }^{\circ} \mathrm{C}$ (Raper y Milles 1958).

El objetivo del presente trabajo fue cuantificar e identificar la disponibilidad de lisina con el empleo de $S$. commune en cultivares de maíz, y su relación con la fertilización nitrogenada, además de la densidad de población.

\section{MATERIALES Y MÉTODOS}

Los granos de maíz QPM, así como los maíces Opaco-2, sintético-blanco y amarillo empleados en esta investigación provienen del CIMMYT, UAAAN y del Instituto Tecnológico de Roque. El trabajo se desarrolló en dos fases: la primera fue en campo y la segunda en los laboratorios de Alimentos del Instituto Tecnológico de Celaya y de Calidad Nutritiva del Instituto Mexicano del Maíz de la UAAAN.

El incremento de semilla de los cultivares se realizó en el campo experimental del Instituto Tecnológico de Roque ubicado en la localidad de Celaya, Gto., a $\operatorname{los} 20^{\circ} 30^{\prime} 28^{\prime \prime}$ de latitud Norte y $100^{\circ} 50$ ' 00" de longitud Oeste, a una altitud de $1.750 \mathrm{msnm}$, durante el ciclo agrícola Primavera-Verano de 2005. El suelo se clasifica como franco arcilloso, no salino, con materia orgánica y nitrógeno medio pobre y $\mathrm{pH}$ neutro.

Se evaluó una variedad sintética de grano blanco, tres variedades de alto contenido de proteina, $\mathrm{QPM}_{1}$ genealogía CIMMYT CMSQ993027, QPM CMSQ993037, QPM CMSQ983051 (cristalino), una variedad sintética de grano amarillo y el Opaco-2; el factor, densidad de población se estableció a niveles de 60, 70 y 80 mil plantas/ha, y la fertilización nitrogenada a $0,120,240$ y $360 \mathrm{~kg}$ de N/ha. La unidad experimental consistió de tres surcos a 0,75 m de separación y $5 \mathrm{~m}$ de largo. Las actividades agrícolas se realizaron bajo la recomendación regional para el cultivo de maíz (CEBAJ 1993). Los tratamientos derivados se establecieron en dos repeticiones y dos de fechas siembra: $1^{\circ}$ de abril y 8 de mayo de 2005 .
En muestras de maíz de cada cultivar, se hicieron las determinaciones químicas, cuantificación de humedad $(930,15)$, grasa $(920,39)$, cenizas $(942,05)$, proteínas $(954,01)$ y la valoración de carbohidratos por diferencia (Anónimo 1993). Para ello, se realizó un análisis por reflectancia en infrarrojo cercano (NIR) ver. 1.50. Para llevar a cabo la prueba, el maíz se trituró y se pasó por una malla \# 80, la muestra ya molida se colocó en celdas propias del equipo y se distribuyó homogéneamente, después la muestra fue transferida al porta-celdas para realizar la lectura. Se registraron en una biblioteca los espectros, la humedad, grasa, ceniza, proteína y carbohidratos.

En granos de maíz de todos los tratamientos se realizó un corte de ambos lados del embrión (granos), dejando el endospermo intacto, posteriormente se tomaron 0,12 g de muestra de cada cultivar, enseguida se colocaron en el tubo de ensayo y una gradilla. Después se agregó $0,5 \mathrm{ml}$ de agua destilada a todos los tubos, se taparon con algodón y se esterilizaron a $120{ }^{\circ} \mathrm{C}$, $20 \mathrm{lb}$ de presión por 20 minutos, después se dejaron enfriar.

Para preparar el medio mínimo para sembrar, se pesaron todos los reactivos, colocándolos en un matraz Erlenmeyer de $1.000 \mathrm{ml}$, incluyendo el agua destilada, excepto la tiamina, se disolvieron con un agitador magnético en el matraz, el cual fue colocado en la parrilla eléctrica, una vez disuelto se agregó la tiamina, y se puso al fuego por cinco minutos. Se esterilizó a 120 ${ }^{\circ} \mathrm{C}, 20 \mathrm{lb}$ de presión por 20 minutos y se dejó enfriar. Previamente desinfectado, se pasó al cuarto de siembra, para agregar el hongo $S$. commune al medio. Dentro del mismo se agregó agua destilada esterilizada, para lavar el hongo, hasta que el agua se encuentra casi limpia. Una vez lavado, se licuó con $125 \mathrm{ml}$ de agua destilada, se agregó al medio, se agitó manualmente hasta obtener una mezcla uniforme. Enseguida se destaparon los tubos, se agregó $2 \mathrm{ml}$ de medio mínimo con los micelios suspendidos. Se incubaron de ocho a 10 días a $28{ }^{\circ} \mathrm{C}$ y se registró el crecimiento de micelios.

Para incrementar el hongo, se pesaron todos los reactivos, se colocaron en un matraz erlenmeyer de $1.000 \mathrm{ml}$, incluyendo el agua destilada, excepto la lisina. Se puso un agitador magnético en el matraz y se colocó en la parrilla eléctrica hasta disolver 
completamente. Una vez disuelto se agregó la lisina y se puso a calentar por cinco minutos. Se esterilizó a 120 ${ }^{\circ} \mathrm{C}, 20 \mathrm{lb}$ de presión por 20 minutos y se dejó enfriar. En el cuarto de siembra, se tomó una pequeña cantidad de hongo (cepa) con un asa y se colocó en el medio, posteriormente, se tapó y se puso a incubar hasta lograr el incremento del hongo.

Una vez que el hongo alcanzó su máximo crecimiento se tomaron las lecturas correspondientes. Para ello se utilizó una escala ya establecida por el método microbiológico utilizado, y se observó cada tubo para darle una calificación de acuerdo al crecimiento que presentó el hongo. Para ello, se utilizó una escala que varía de 0 a 5 , en intervalos de 0,5 ; los no seleccionados van de 0 hasta 2,0 , los seleccionados de 2,5 a 3,5 y los sobre seleccionados de 4,0 a 5,0; los no seleccionados tienen un bajo contenido de lisina, los seleccionados tienen un alto contenido de lisina y los sobre seleccionados tienen muy alto contenido de lisina.

En campo se utilizó un diseño experimental de bloques completos al azar con arreglo de parcelas subdivididas. En la parcela grande se asignaron las variedades, en la mediana a las densidades y en la chica se ubicó la fertilización nitrogenada. Para el estudio de laboratorio se aplicó un diseño completamente al azar, además, se realizó la prueba de comparación de medias Duncan a $\alpha=0,05$ (Little y Hills 1981), con el paquete estadístico SAS ver. 6,12 (SAS 1989).

\section{RESULTADOS Y DISCUSIÓN}

En los datos del análisis de varianza existieron diferencias estadísticas altamente significativas $(\mathrm{P} \geq 0,01)$ en la fuente de variación variedades o genotipos, fertilización y la interacción variedad*fertilización, diferencias al 5\% de probabilidad para densidad e interacción densidad*fertilización para la variable disponibilidad de lisina. La proteína total en el grano fue significativa para variedades $(\mathrm{P} \geq 0,05)$, no existió tal diferencia para contenido de grasa, ceniza y carbohidratos. En el análisis del germen se encontró diferencia significativa en la proteína, ceniza y carbohidratos. En el endospermo, la proteína y la ceniza fueron diferentes significativamente al 5\% entre variedades (Cuadro 1). Para la característica de rendimiento el factor variedad, densidad y fertilización influyeron en la respuesta entre tratamientos. Esto significa que los niveles incluidos en cada factor fueron determinantes en los resultados anteriores y la variabilidad observada se atribuye tanto a la genética del individuo como al factor ambiental, por lo tanto, ésto puede influir en la distribución ponderal y en la composición química del grano, endospermo y germen.

Cuadro 1. Comparación de medias para los macro-componentes de variedades de maíz QPM y normal. Técnica en Infrarrojo (NIR). Universidad Autónoma Agraria Antonio Narro (UAAAN), México. 2005.

\begin{tabular}{lcccccc}
\hline & & & & \multicolumn{2}{c}{ G de lisina/ 100 g de proteína } \\
\cline { 5 - 7 } \multicolumn{1}{c}{ Variedad } & Proteína & Proteína & Proteína & Grano & Endospermo & Germen \\
& total & germen & endospermo & entero & & \\
\hline VS* (V1) & $11,08 \mathrm{a}$ & $18,73 \mathrm{~d}$ & $10,36 \mathrm{a}$ & $2,05 \mathrm{c}$ & $1,69 \mathrm{c}$ & $7,73 \mathrm{a}$ \\
QPM1 & $10,68 \mathrm{ab}$ & $20,52 \mathrm{bc}$ & $9,07 \mathrm{~b}$ & $3,75 \mathrm{a}$ & $4,13 \mathrm{a}$ & $5,62 \mathrm{~b}$ \\
QPM2 & $10,50 \mathrm{ab}$ & $21,29 \mathrm{a}$ & $9,95 \mathrm{ab}$ & $3,58 \mathrm{ab}$ & $2,41 \mathrm{~b}$ & $5,48 \mathrm{bc}$ \\
Amarillo (V2) & $10,78 \mathrm{a}$ & $20,47 \mathrm{bc}$ & $9,41 \mathrm{~b}$ & $1,64 \mathrm{c}$ & $1,91 \mathrm{bc}$ & $5,68 \mathrm{~b}$ \\
Cristalino (QPM3) & $10,40 \mathrm{ab}$ & $20,91 \mathrm{ab}$ & $9,26 \mathrm{~b}$ & $2,46 \mathrm{bc}$ & $4,01 \mathrm{a}$ & $4,78 \mathrm{c}$ \\
Media & 10,68 & 20,38 & 9,61 & 2,69 & 2,83 & 5,85 \\
Opaco 2** & & 35,10 & 60,70 & & 18,89 & 79,10 \\
DMS & 1,01 & 0,62 & 0,91 & 1,27 & 0,66 & 0,80 \\
\hline
\end{tabular}

* $\mathrm{V}_{1}$ y $\mathrm{V}_{4}=$ Testigos de maíz normal blanco y amarillo, respectivamente. **, datos tomados de Landry y Moureaux (1980). 
En este estudio por la técnica en infrarrojo se encontró pequeña variación en el contenido total de proteína en el grano, entre cultivares de alta calidad proteica y normales, los datos variaron de $10,40 \%$ a $11,08 \%$, resultados que coinciden a los reportados por Landry y Moureaux (1982). En el germen y endospermo se encontró una composición que varió de $18,73 \%$ a $21,29 \%$ y $9,04 \%$ a $10,36 \%$, respectivamente, sin embargo, para el cultivar Opaco-2, se reportó 35,1\% de proteína en el germen y 60,7\% en el endospermo, evidencias que concuerdan con Poey (1978) y FAO (1993). Es importante señalar que la alta calidad proteica reportada en el genotipo Opaco-2 se debe a un gene que forma parte de su genoma.

Si la calidad nutritiva del maíz está determinada por la composición de aminoácidos (proteínas) y se observa amplia variación entre tratamientos, es necesario trabajar con el aspecto genético, ambiental y manejo agronómico, debido a que estos factores pueden influir en la distribución ponderal, en la composición química específica del endospermo y germen del maíz (Bressani et al. 1990). Cabe señalar, que después del almidón, la proteína constituye el siguiente componente químico del grano por orden de importancia (Landry y Moureaux 1982). Es importante mencionar que la calidad de la proteína total del grano es superior en los maíces de endospermo normal como se describe en este experimento, resultados que coinciden con Poey et al. (1979).

Numerosos estudios han analizado la causa de la baja calidad de las proteínas del maíz, asimismo, se ha demostrado que al agregar lisina al maíz se mejora levemente la calidad de las proteínas. En este estudio se observa que la cantidad de lisina fue superior en aquellos cultivares QPM's que son de alta calidad proteica que los normales blancos y amarillos. A través de los genotipos, se puede demostrar que la cantidad de proteina es mayor en el germen que la disponible en el endospermo. Los valores medios de los cultivares son 2,83 y 5,85 g de lisina/100 g de proteína para endospermo y germen, respectivamente. Esta variación se puede aprovechar en un programa de mejoramiento genético, además el maíz Opaco-2 y el maíz QPM son agronómicamente mejores a los que actualmente se encuentran en el mercado. Resultados satisfactorios en México y otros países, donde se comercializan cultivares QPM's con rendimientos similares a los maíces de endospermo normal (Anónimo 2005), indican que se puede mejorar simultáneamente la calidad y el rendimiento de los cultivos.

$\mathrm{Al}$ analizar cada una de las variables en estudio se puede demostrar primero, que el hongo S. commune permitió determinar y cuantificar la presencia de lisina en los diferentes cultivares. Así se puede mencionar, derivado de estos resultados, que los valores de lisina difirieron entre las variedades, con valores que variaron del $17,4 \%$ de lisina que pertenece a la variedad sintética normal (V1) hasta el 58,7\% que mostró el cultivar Opaco-2. Entre la variedad sintética, el QPM $\mathrm{QPM}_{2}, \mathrm{QPM}_{3}$, maíz amarillo y cristalino la variación fue pequeña para esta variable $(4,3 \%)$. La prueba de comparación de medias arrojó un valor DMS de 2,003\% (Figura 1).

La presencia de lisina fue afectada significativamente $(\mathrm{P} \leq 0,01)$ por la densidad de población, es decir, a medida que la población de plantas por hectárea se incrementó, la cantidad de lisina disminuyó. A 60.000 p/ha se encontró $26,5 \%$, a 70.000 p/ha $18,8 \%$ y a 80.000 p/ha 17,6\% (Figura 2). Algunos estudios describen que el incremento de la densidad de población conduce a una disminución en el uso eficiente de la radiación solar (fotosíntesis) (Purcell et al. 2002), el cual puede afectar significativamente a la producción y calidad del grano del maíz. Otros trabajos de Widdicombe y Thelen (2002) demuestran que el mayor rendimiento de maíz se tiene con densidades de 90.000 p/ha, aunque también destacó la interacción densidad

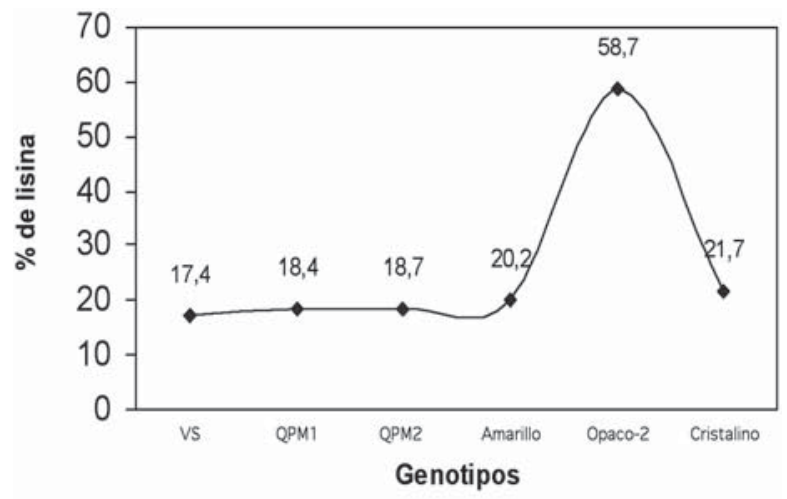

Figura 1. Disponibilidad de lisina en granos de maíz provenientes de diferente origen. Lisina determinada por Schizophyllum commune. Universidad Autónoma Agraria Antonio Narro (UAAAN), México. 2005. 


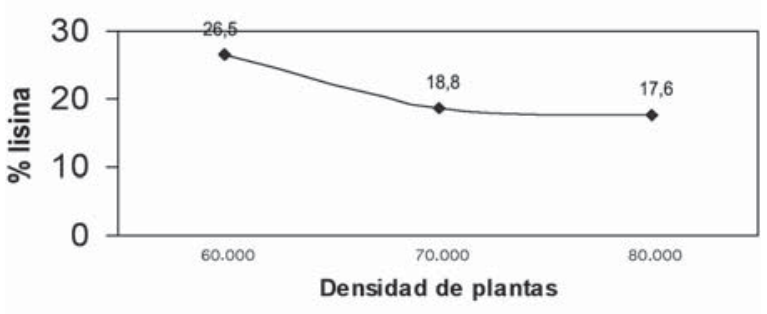

Figura 2. Respuesta del contenido de lisina en granos de maíz a la densidad de población. Lisina determinada por Schizophyllum commune. Universidad Autónoma Agraria Antonio Narro (UAAAN), México. 2005.

por genotipo. Mencionan que las densidades de población altas disminuyen la eficiencia de uso de la radiación que, junto con la ausencia de efectos estadísticos respecto a la aplicación de $\mathrm{N}$, pudo haber decrecido el nitrógeno foliar $\mathrm{y}$, como consecuencia, la producción y la misma calidad del grano.

La fertilización con base a nitrógeno también fue motivo de variación para el contenido de lisina, entre 0 y 120 unidades de $\mathrm{N}$ produjeron entre 31,4\% y 33,6 $\%$ de lisina. Pero la presencia de lisina se redujo significativamente a $9,77 \%$ y $12,29 \%$ con la adición de 240 y 360 unidades de N, respectivamente. Lo anterior indica que esta variable es fuertemente influenciada por la estructura genética de los individuos, factores ambientales, pero más, a la densidad de población y fertilización nitrogenada (Figura 3). Si bien esta calidad es una respuesta intrínseca de la planta, la adición de nitrógeno puede contribuir a ella de manera importante, pues actúa como componente de vitaminas y diversos sistemas de energía en la planta; también forma parte de aminoácidos, precursores de proteínas,

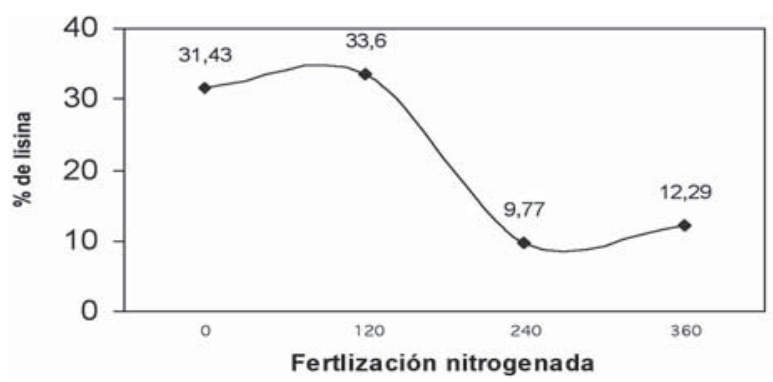

Figura 3. Respuesta del contenido de lisina en granos de maíz a la fertilización nitrogenada. Lisina determinada por Schizophyllum commune. Universidad Autónoma Agraria Antonio Narro (UAAAN), México. 2005. ácidos nucléicos, aminas, amidas, nucleoproteínas y de la clorofila (Domínguez 1997).

\section{CONCLUSIONES}

Los resultados indican que el hongo Schizophyllum commune permitió determinar la presencia de la lisina en los diferentes cultivares. El contenido total de proteína en el grano entre cultivares de alta calidad proteica y normales varió de $10,40 \%$ a $11,0 \%$. La composición en el germen y endospermo varió de $18,73 \%$ a $21,29 \%$ y $9,04 \%$ a $10,36 \%$, respectivamente. Para el cultivar Opaco-2, se encontró 58,7\% de lisina. Los valores para lisina fueron superiores en los maíces QPM's versus grano blanco. El porciento de lisina fue fuertemente influenciada por el genotipo, la densidad de población y fertilización nitrogenada. El mayor contenido de lisina se encontró a 120 unidades de $\mathrm{N}$ y a 60.000 p/ha.

\section{LITERATURA CITADA}

ANÓNIMO. 1993. El maíz en la nutrición humana. Alimentación y Nutrición No. 25. La ONU para la Agricultura y la Alimentación FAO. Roma. 114 p.

ANÓNIMO. 2005. Maíz de alta calidad proteínica. (en línea). México, D.F. Consultado 8 enero 2005. Disponible en: http:www.inifap.conacyt.mx

BRESSANI, R.; BENAVIDES, V.; ACEVEDO, E.; ORTIZ, M.A. 1990. Changes in selected nutrient contents and in protein quality of common and quality-protein maize during tortilla preparation. Cereal Chem. 67(6): 515-518.

CEBAJ. CENTRO EXPERIMENTAL BAJÍO. 1993. Guía para cultivar maíz de riego y temporal en Guanajuato. Boletín Informativo. Instituto Nacional de Investigaciones Forestales, Agrícolas y Pecuarias. Celaya, Guanajuato, México. Edición INIFAP. 36 p.

CHÁVEZ, J. F. 1973. El maíz en la nutrición de México. En simposio sobre desarrollo y utilización de maíces de alto valor nutritivo. (1972, México D.F.). Memoria Colegio de posgraduados ENA. Chapingo, México. p. 9-11.

AGRONOMÍA MESOAMERICANA 18(2): 177-183. 2007 
CLARIDADES. 2006. Revista Claridades Agropecuarias no. 157. Septiembre 2006. Editada por Apoyos y Servicios a la comercialización (ASERCA). México, D. F. p. 16-41.

DOMÍNGUEZ, V. A. 1997. Tratado de fertilización. 3 ed. Mundi-Prensa. México, D. F., México. 613 p.

LANDRY, J.; MOUREAUX, T. 1982. Distribution and amino acid composition of protein fractions in opaque-2 maize grain. Phytochemistry 21: $1865-1869$.

LITTLE, T. M.; HILLS, F. J. 1981. Métodos estadísticos para la investigación en la agricultura. Traducido de la segunda reimpresión en inglés por Crespo, A. P. Tercera reimpresión. Editorial Trillas, México, D.F. 270 p.

POEY, D. F. R. 1979. Mejoramiento integral del maíz: rendimiento y valor nutritivo; hipótesis y métodos. Tesis Doctoral, Colegio de Posgraduados, Chapingo, Estado de México, México. 206 p.
PURCELL, L. C.; BALL R. A.; REAPER, J. D.; VORIES, E. D. 2002. Radiation use efficiency and biomass production in soybean at different plant population densities. Crop Sci. 42:172-177.

RAPER, J. R.; MILES, P. C. 1958. The genetics of Schizophyllum commune. Genetics 43(1):530-546.

SAS, Institute INC. 1989. SAS/OR User's Guide Versión 6.12. Cary, NC: SAS Institute. 479 p.

WALL, J. S.; PAULIS, J. W. 1985. Evaluación química y biológica de la calidad proteínica del maíz: Asuntos y problemas actuales. In: Maíz de alta calidad proteínica. (1985. CIMMYT, México, D.F.). Memoria México. p. 305-313.

WIDDICOMBE, W. D.; THELEN, K. D. 2002. Row width and plant density effects on corn grain production in the northern corn belt. Agron. J. 94:1020-1023. 